\title{
A Lopsided Merger
}

\author{
The merger of two black holes with significantly different masses allows \\ researchers to better characterize black hole parameters and to perform \\ new tests of general relativity.
}

\author{
By Stephen Taylor
}

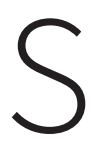
ince LIGO and Virgo first observed the merger of two black holes in September 2015 [1] (see Viewpoint: The First Sounds of Merging Black Holes), gravitational-wave detection has become a regular occurrence. Yet the warped Universe continues to yield a bounty of diverse discoveries. Now, the LIGO and Virgo collaborations report that, just two weeks into their third observation run, the LIGO and Virgo detectors spotted a black hole merger, dubbed GW190412, that was remarkably different from those previously measured: With 8 and 30 solar masses, respectively, this "Laurel \& Hardy" duo is the first truly asymmetric black hole binary system ever spotted (Fig. 1) [2]. The detection of such an asymmetric binary has allowed a trove of new scientific possibilities, from placing strong constraints on the black holes' spins, to suggesting new astrophysical scenarios for the formation of such lopsided systems, to testing Einstein's theory of general relativity in previously unexplored regimes.

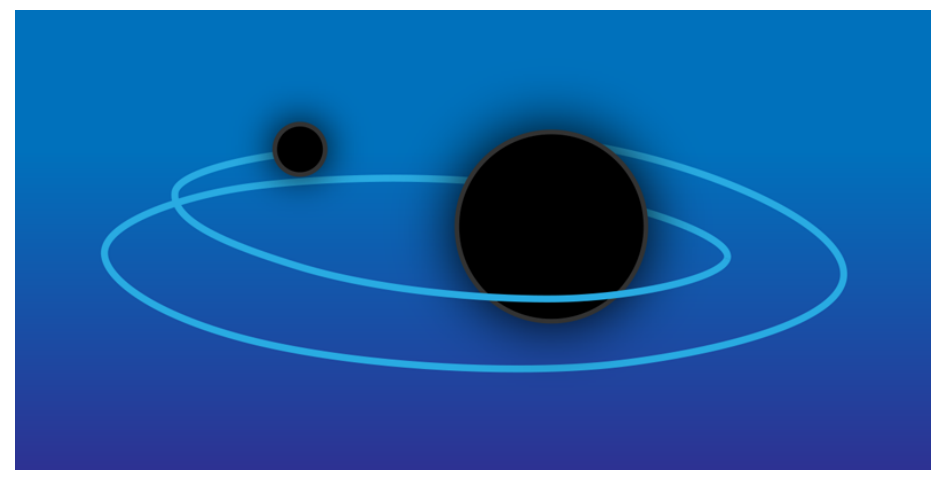

Figure 1: The LIGO and Virgo detectors have spotted the merger of a remarkably asymmetric black hole duo, whose components weigh 8 and 30 solar masses, respectively.

Credit: APS/Alan Stonebraker
Gravitational waves naturally arise in the theory of general relativity, which connects gravity to the warping of spacetime in the presence of matter. A pair of compact objects like neutron stars or black holes churn up spacetime as they orbit one another, inspiral, and eventually merge, creating spacetime ripples that radiate outwards at the speed of light. Billions of years later, the waves reach our gravitational-wave detectors, where they slightly deform the detector's orthogonal, kilometers-long arms. Such a pattern of deformations encodes the merging system's dynamics, including the orbital geometry and the properties of each neutron star or black hole.

Until now, most detections showed pairs of black holes with roughly comparable masses: Even the most asymmetric detected mergers involved mass ratios of less than 2 [3]. As predicted by general relativity, the gravitational-wave signal in these symmetric cases is dominated by a single frequency-the second harmonic of the binary orbital frequency [4].

Furthermore, the signals from all detections but two (GW151226 and GW170729) have been consistent with the binary having an effective spin of zero. This parameter is a mass-weighted sum of each black hole's spin component perpendicular to the orbital plane. A vanishing effective spin for same-weight black holes means that the two bodies either aren't spinning or they are spinning in opposite directions. A nonzero effective spin would significantly affect the merger dynamics.

At first glance, GW190412 is in line with previous discoveries-it is a pair of black holes with individual masses consistent with previous detections and with the black hole formation pathways considered by theory. At the same time, the asymmetry of the binary's two masses makes it a beautifully divergent system compared to anything seen before (Video 1). LIGO and Virgo used a variety of different waveform models to 


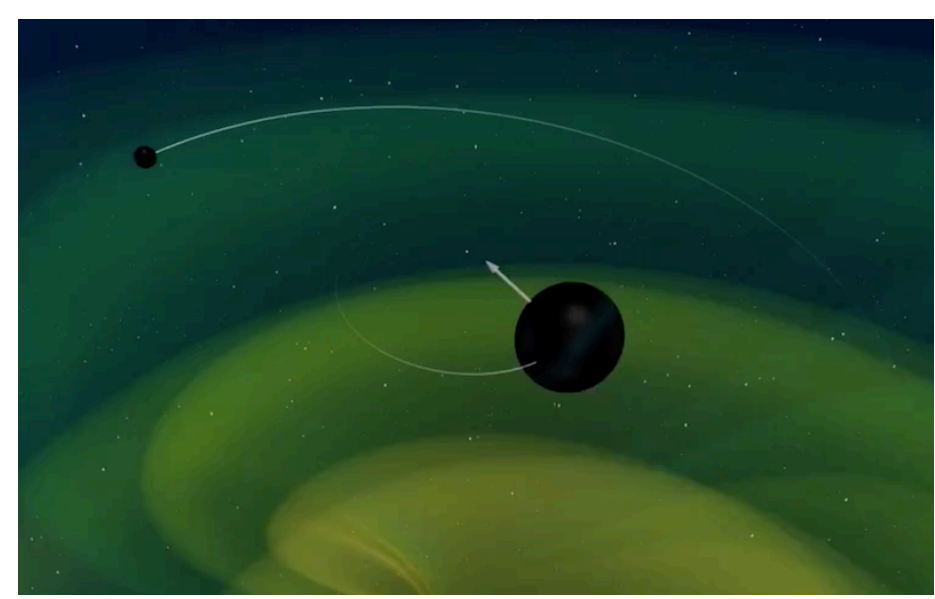

Video 1: Numerical simulations of the inspiral and merger of two black holes with a mass ratio of 3.5 are consistent with the GW190412 observation.

Credit: N. Fischer, H. Pfeiffer, A. Buonanno/Max Planck Institute for Gravitational Physics/Simulating eXtreme Spacetimes project

determine that one of the black holes was about 3.5 times as massive as the other. In accord with general relativity, this asymmetry means that, in addition to the main second-harmonic emission frequency, higher overtones of gravitational-wave emission, specifically the mode at 3 times the binary orbital frequency, were detectable. Gravitational waves have often been referred to as the music of the cosmos, and in this case the analogy is quite apt: The collision of these black holes out in the vastness of the Universe produced a signal containing a "perfect fifth"-a musical interval, like that between the $\mathrm{G}$ and $\mathrm{C}$ notes, corresponding to two frequencies with a $3: 2$ ratio [5].

The lopsided black hole duo allowed the researchers to vet general relativity in previously unexplored regimes. Namely, by testing the theory's predictions for the multipole moments associated with the higher-harmonic emissions of an asymmetric merger. All parameters associated with deviations from general relativity were consistent with being zero-even as the nuances of his theory are tested in novel ways, Einstein continues to be correct.

By fitting the more complex signal containing the higher overtones, the researchers drastically improved, compared to previous detections, constraints on system parameters such as the binary's distance from Earth, its mass ratio, and the black holes' spin. In fact, the LIGO and Virgo scientists report the most precise determination of a black hole spin ever extracted from gravitational-wave data, finding that the larger black hole's event horizon spins at about $43 \%$ of the speed of light.

GW190412 is now the third signal to show evidence of a nonzero spin $[6,7]$, showing that there is great potential to determine this feature cleanly and directly using gravitational-wave analysis. Other common spin characterization techniques are indirect, as they infer a black hole's spin from the x-ray emission of material falling into the black hole and must thus rely on hard-to-test models of accretion dynamics. For GW190412, the signal analysis also hinted at a difficult-to-observe effect: a mild precession of the orbital spins suggesting that the spins of each black hole were not aligned with the axis of the orbital motion.

GW190412's peculiarity among its peers-the measurable spin and the asymmetric masses-makes it a valuable addition that informs black hole demographics. From previously detected mergers, researchers showed that the probability of finding black hole binaries with certain sizes can be described by a power law. The inclusion of this asymmetric duo yields much tighter constraints on the distribution of expected mass ratios. While previous analysis suggests that a system at least as asymmetric as GW190412 should only arise in $1.7 \%$ of the cases [8], the population distribution law that accounts for the new detection revises that probability to $25 \%$.

The detection of GW190412 shows just how much more we have to learn from merging black holes. An important result would be the extraction of even higher overtones. By breaking important parameter degeneracies, such overtones would improve measurements of the distances of black hole binaries, allowing them to be used as "dark sirens" for inferring the local expansion rate of the Universe [9]. This approach could help researchers settle the debate over the Hubble constant-for which cosmic microwave background and supernovae measurements deliver conflicting values (see Feature: Cosmologists Can't Agree on the Hubble Constant). And, detections of other asymmetric systems and their spins may shed light on possible formation channels. The misaligned spins of the GW190412 black holes may suggest, for instance, that the duo doesn't come directly from a stellar binary. Instead, one of them could be the product of a previous black 
hole merger [10]. A better characterization of black hole spins and of the environment in which the merger took place could deliver conclusive evidence for this hypothesis. Surely, we can expect that upcoming detections will deliver many more insights into the smorgasbord of scenarios that form coalescing black hole pairs. Indeed, during the writing of this Viewpoint, researchers reported an even more asymmetric system, called GW190814, which hosts either the heaviest neutron star or the lightest black hole ever discovered [11].

Stephen Taylor: Department of Physics and Astronomy, Vanderbilt University, Nashville TN, USA

\section{REFERENCES}

1. B. P. Abbott et al. (LIGO Scientific Collaboration and Virgo Collaboration), "Observation of gravitational waves from a binary black hole merger,” Phys. Rev. Lett. 116, 061102 (2016).

2. R. Abbott et al. (LIGO Scientific Collaboration and Virgo Collaboration), "GW190412: Observation of a binary-black-hole coalescence with asymmetric masses," Phys. Rev. D 102, 043015 (2020).

3. B. P. Abbott et al. (LIGO Scientific Collaboration and Virgo Collaboration), "Binary black hole population properties inferred from the first and second observing runs of advanced LIGO and advanced Virgo," Astrophys. J. 882, L24 (2019).

4. L. Blanchet, "Gravitational radiation from post-Newtonian sources and inspiralling compact binaries," Living Rev. Relativ. 17, 2 (2014).

5. C. Berry, "GW190412-A new flavour of binary black hole," http s://cplberry.com/2020/04/18/gw190412.

6. B. P. Abbott et al. (LIGO Scientific Collaboration and Virgo Collaboration), "GW151226: Observation of gravitational waves from a 22-solar-mass binary black hole coalescence," Phys. Rev. Lett. 116, 241103 (2016).

7. K. Chatziioannou et al., "On the properties of the massive binary black hole merger GW170729," Phys. Rev. D 100, 104015 (2019).

8. B. P. Abbott et al. (LIGO Scientific Collaboration and Virgo Collaboration), "GWTC-1: A gravitational-wave transient catalog of compact binary mergers observed by LIGO and Virgo during the first and second observing runs," Phys. Rev. X 9, 031040 (2019).

9. M. Soares-Santos et al. (LIGO Scientific Collaboration and Virgo Collaboration), "First measurement of the hubble constant from a dark standard siren using the dark energy survey galaxies and the LIGO/Virgo binary-black-hole merger GW170814," Astrophys. J. 876, L7 (2019).

10. D. Gerosa, "Astrophysical implications of GW190412 as a remnant of a previous black-hole merger," arXiv:2005.04243.

11. R. Abbott et al. (LIGO Scientific Collaboration and Virgo Collaboration), "GW190814: Gravitational waves from the coalescence of a 23 solar mass black hole with a 2.6 solar mass compact object," Astrophy. J. 896, L44 (2020). 